Journal of Computational and Applied Mechanics, Vol. 14, No. 1-2, (2019), pp. 41-51 DOI: $10.32973 /$ jcam.2019.003

\title{
ANALYSIS OF A CURVED BIMETALLIC BEAM
}

\author{
DÁVID GÖNCZI \\ Institute of Applied Mechanics, University of Miskolc \\ H-3515 Miskolc-Egyetemváros, Hungary \\ mechgoda@uni-miskolc.hu
}

[Received: December 12, 2018; Accepted: April 26, 2019]

\begin{abstract}
This paper deals with the determination of stresses and displacements in a curved bimetallic beam which has uniform curvature. The two curved beam components of different materials have common displacements at their interface. The thermal load is derived from uniform temperature change. Two models are considered. The first one is based on the theory of the generalized plane stress state of elasticity and the second one uses a strength of materials approach. The results obtained by these models are verified by a comparison with finite element analysis.
\end{abstract}

Mathematical Subject Classification: 74B05, 34B05

Keywords: Bimetallic, curved beam, uniform temperature change, stresses, displacement, FEM

\section{INTRODUCTION}

When two metal strips, strip 1 and strip 2, are bounded along the length of a beam, strip 1 is partly prevented from expanding by strip 2 when they are subjected to uniform temperature change. A considerable force is thereby developed which causes the bonded strip to bend. If there are no applied external forces, the bimetallic strip will take the shape of a circular arc. Timoshenko [1] was the first who studied stresses in bimetallic beams. He used the expressions of curvature and stresses assuming that the temperature change is uniform [1-3]. Several works have dealt with the analysis of bimetallic strips which consist of two different beam components $4-10$. In the above-mentioned papers curved bimetallic beams were not considered. In papers 11 , 12 curved beams are investigated but the loading is pure mechanical loading. In this paper the curved bimetallic beam under the action of uniform temperature change is studied. The considered bimetallic beam has uniform curvature. To determine the deformations and stresses in curved bimetallic beams two methods will be considered. The first one is an elasticity solution based on the theory of generalized plane stress state. The second approach uses a strength of materials formulation. The results obtained by these models are verified by comparison with a finite element solution (Abaqus CAE). 


\section{Solution as a Generalized Plane Stress PROBlem}

Figure 1 shows the curved bimetallic beam of uniform curvature, which consists of two different elastic materials.

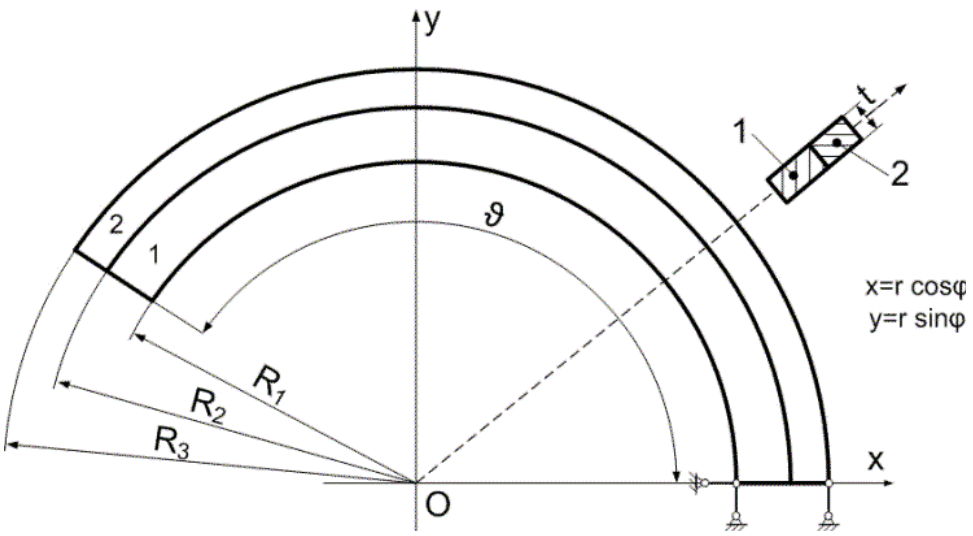

Figure 1. Bimetallic curved beam with rectangular cross section

The governing equations and boundary conditions are formulated in the cylindrical coordinate system $\operatorname{Or} \varphi z$, and the plane $z=0$ is the symmetry plane of the twolayered curved beam for its geometrical properties. The connection between beam component 1 and beam component 2 at the common cylindrical boundary surface $r=R_{2}$ is perfect, hence neither the displacements nor the tractions have a jump at $r=R_{2}$. For example, technically the rigid connection between the beams 1 and 2 is created by welded bonds. There are no present body forces and the whole boundary of the bimetallic curved beam is stress free. This means that

$$
\begin{gathered}
\sigma_{r}\left(R_{1}, \varphi\right)=\sigma_{r}\left(R_{3}, \varphi\right)=\tau_{r \varphi}\left(R_{1}, \varphi\right)=\tau_{r \varphi}\left(R_{3}, \varphi\right)=0, \quad 0 \leq \varphi \leq \vartheta, \\
\sigma_{\varphi}(r, \vartheta)=\sigma_{\varphi}(r, 0)=\tau_{r \varphi}(r, \vartheta)=\tau_{r \varphi}(r, 0)=0, \quad R_{1} \leq r \leq R_{3} .
\end{gathered}
$$

In the framework of generalized plane stress model the boundary condition

$$
\sigma_{\varphi}(r, \vartheta)=\sigma_{\varphi}(r, 0)=0, \quad R_{1} \leq r \leq R_{3},
$$

will be satisfied only in weak form such as

$$
\begin{gathered}
N=\int_{R_{1}}^{R_{3}} \sigma_{\varphi}(r, \vartheta) \mathrm{d} r=\int_{R_{1}}^{R_{3}} \sigma_{\varphi}(r, 0) \mathrm{d} r=0, \\
M=\int_{R_{1}}^{R_{3}} r \sigma_{\varphi}(r, \vartheta) \mathrm{d} r=\int_{R_{1}}^{R_{3}} r \sigma_{\varphi}(r, 0) \mathrm{d} r=0 .
\end{gathered}
$$

That is, the stress resultants and the moment resultant of the stresses vanish only at the end cross sections $\varphi=0$ and $\varphi=\vartheta$. The temperature of the two-layer composite beam initially is the reference temperature. Its temperature is slowly raised to a 
constant uniform temperature, where the temperature change is $T$. The deformations and stresses are caused by only the uniform change of temperature. The solution of this problem is derived from the following displacement field

$$
\begin{gathered}
u_{i}(r, \varphi)=U_{i}(r)+f_{1} \cos \varphi+f_{2} \sin \varphi, \quad(i=1,2), \\
v_{i}(r, \varphi)=C r \varphi+f_{1} \sin \varphi-f_{2} \cos \varphi+f_{3} r, \quad(i=1,2),
\end{gathered}
$$

where the displacement components are denoted by $u_{i}$ in the radial direction and by $v_{i}$ in the circumferential direction and the lower index $i$ refers to curved beam component $(i=1,2)$. In equations (6), (7) $f_{1}, f_{2}$ and $f_{3}$ are integration constants whose values can be obtained from the displacement boundary conditions (Figure 1):

$$
u_{1}\left(R_{1}, 0\right)=0, \quad v_{1}\left(R_{1}, 0\right)=0, \quad v_{2}\left(R_{3}, 0\right)=0 .
$$

The constant $C$ can be determined from the stress boundary conditions (1), (4), (5) and the continuity conditions the displacements and the normal stress field should satisfy on the common cylindrical boundary of the beam components 1 and 2 . It follows from equations (6) and (7) that the strains can be given in terms of $U_{i}$ and $C$ as

$$
\varepsilon_{\varphi i}=\frac{U_{i}}{r}+C, \quad \varepsilon_{r i}=\frac{d U_{i}}{d r}, \quad(i=1,2) .
$$

Combining equations $(9)_{1}$ and $(9)_{1}$ yields the compatibility condition the strains should meet:

$$
r \frac{d \varepsilon_{\varphi i}}{d r}+\varepsilon_{\varphi i}-\varepsilon_{r i}-C=0, \quad(i=1,2) .
$$

For the present problem the constitutive law of linear thermoelasticity has the following form 9, 10:

$$
\begin{array}{ll}
E_{i} \varepsilon_{r i}=\sigma_{r i}-\nu_{i} \sigma_{\varphi i}+E_{i} \alpha_{i} T, & (i=1,2), \\
E_{i} \varepsilon_{\varphi i}=\sigma_{\varphi i}-\nu_{i} \sigma_{r i}+E_{i} \alpha_{i} T, & (i=1,2),
\end{array}
$$

where $E_{i}$ is the modulus of elasticity, $\nu_{i}$ is the Poisson ratio and $\alpha_{i}$ is the coefficient of linear thermal expansion while the radial and circumferential normal stresses are denoted by $\sigma_{r i}$ and $\sigma_{\varphi i}(i=1,2)$. Substituting equations (11) and 12 into (10) yields

$$
r \frac{d}{d r}\left(-\nu_{i} \sigma_{r i}+\sigma_{\varphi i}+E_{i} \alpha_{i} T\right)-\left(1+\nu_{i}\right) \sigma_{r i}+\left(1+\nu_{i}\right) \sigma_{\varphi i}-E_{i} C=0, \quad(i=1,2) .
$$

In our case the equation of mechanical equilibrium is as follows:

$$
\frac{\mathrm{d} \sigma_{r i}}{\mathrm{~d} r}+\frac{\sigma_{r i}-\sigma_{\varphi i}}{r}=0, \quad(i=1,2) .
$$

The general solution of equation (14) can be given in terms of the stress functions $F_{i}=F_{i}(r)$ as

$$
\sigma_{r i}=\frac{F_{i}(r)}{r}, \quad \sigma_{\varphi i}=\frac{\mathrm{d} F_{i}}{\mathrm{~d} r}, \quad(i=1,2) .
$$

Combination of equation 113 with the formulas derived for the normal stresses leads to the following differential equations

$$
r^{2} \frac{\mathrm{d} F_{i}^{2}}{\mathrm{~d}^{2} r}+r \frac{\mathrm{d} F_{i}}{\mathrm{~d} r}-F_{i}-E_{i} C r=0, \quad(i=1,2),
$$


the solution of which are given by

$$
F_{1}(r)=c_{1} r+\frac{c_{2}}{r}+\frac{E_{1} C}{2} r \ln r, \quad R_{1} \leq r<R_{2},
$$

and

$$
F_{2}(r)=c_{3} r+\frac{c_{4}}{r}+\frac{E_{2} C}{2} r \ln r, \quad R_{2}<r \leq R_{3},
$$

where $c_{1}, c_{2}, c_{3}$ and $c_{4}$ are undetermined integration constants. Note that the stress and strain fields are independent of the polar angle $\varphi$. A simple calculation shows that

$$
\begin{aligned}
N=\int_{R_{1}}^{R_{3}} \sigma_{\varphi}(r) \mathrm{d} r= & \int_{R_{1}}^{R_{3}} \frac{\mathrm{d}}{\mathrm{d} r}\left(r \sigma_{r}\right) \mathrm{d} r= \\
& =R_{2} \sigma_{r 1}\left(R_{2}\right)-R_{1} \sigma_{r 1}\left(R_{1}\right)+R_{3} \sigma_{r 2}\left(R_{3}\right)-R_{2} \sigma_{r 2}\left(R_{2}\right)=0
\end{aligned}
$$

if the stress boundary conditions

$$
\sigma_{r 1}\left(R_{1}\right)=\sigma_{r 2}\left(R_{3}\right)=0
$$

and the continuity conditions

$$
\sigma_{r 1}\left(R_{2}\right)=\sigma_{r 2}\left(R_{2}\right)
$$

are satisfied. To obtain the stress field we should determine the five undetermined integration constants $c_{1}, c_{2}, c_{3} c_{4}$ and $C$. The following equations will be used for determining the values of the unknown integration constants:

$$
\begin{gathered}
\sigma_{r 1}\left(R_{1}\right)=0, \quad \sigma_{r 2}\left(R_{3}\right)=0, \quad \sigma_{r 1}\left(R_{2}\right)=\sigma_{r 2}\left(R_{2}\right), \\
U_{1}\left(R_{2}\right)=U_{2}\left(R_{2}\right), \quad \int_{R_{1}}^{R_{2}} r \sigma_{\varphi 1} \mathrm{~d} r+\int_{R_{2}}^{R_{3}} r \sigma_{\varphi 2} \mathrm{~d} r=0 .
\end{gathered}
$$

By the use of $(9)_{1}$ equation 23$)_{1}$ can be manipulated into the following form:

$$
\varepsilon_{\varphi 1}\left(R_{2}\right)=\varepsilon_{\varphi 2}\left(R_{2}\right) .
$$

This equation can be expressed in terms of stresses as

$$
\frac{1}{E_{1}}\left[\sigma_{\varphi 1}\left(R_{2}\right)-\nu_{1} \sigma_{r 1}\left(R_{2}\right)\right]+\alpha_{1} T=\frac{1}{E_{2}}\left[\sigma_{\varphi 2}\left(R_{2}\right)-\nu_{2} \sigma_{r 2}\left(R_{2}\right)\right]+\alpha_{2} T .
$$

By utilizing equations $(22),(23)$ and $(25)$ the following system of linear equations can be set up for $\mathbf{x}^{T}=\left[\begin{array}{l|l|l|l|l}c_{1} & c_{2} & c_{3} & c_{4} & C\end{array}\right]$ :

$$
\mathbf{M x}=\mathbf{h}
$$

where

$$
\underset{(5 \times 5)}{\mathbf{M}}=\left[m_{i j}\right] ; \quad \mathbf{h}^{T}=[0|0| 0|h| 0]
$$

and 


$$
\begin{gathered}
\left.m_{11}=R_{1}, \quad m_{12}=\frac{1}{R_{1}}, m_{13}=m_{14}=m_{21}=m_{22}=0, m_{15}=\frac{E_{1}}{2} R_{1} \ln R_{1}\right) \\
m_{23}=R_{3}, m_{24}=\frac{1}{R_{3}}, m_{25}=\frac{E_{2}}{2} R_{3} \ln R_{3}, \\
m_{31}=R_{2}, m_{32}=\frac{1}{R_{2}}, m_{33}=-R_{2}, m_{34}=-\frac{1}{R_{2}}, m_{35}=\frac{E_{1}-E_{2}}{2} R_{2} \ln R_{2}, \\
m_{41}=\frac{1-\nu_{1}}{E_{1}}, m_{42}=-\frac{1+\nu_{1}}{E_{1} R_{2}^{2}}, m_{43}=-\frac{1-\nu_{2}}{E_{2}}, \\
m_{44}=\frac{1+\nu_{2}}{E_{2} R_{2}^{2}}, m_{45}=\frac{\nu_{2}-\nu_{1}}{2} \ln R_{2}, \\
m_{51}=\frac{1}{2}\left(R_{2}^{2}-R_{1}^{2}\right), m_{52}=-\ln \frac{R_{2}}{R_{1}}, m_{53}=\frac{1}{2}\left(R_{3}^{2}-R_{2}^{2}\right), \\
m_{54}=-\ln \frac{R_{3}}{R_{2}}, h=\left(\alpha_{2}-\alpha_{1}\right) T, \\
m_{55}=\frac{E_{1}}{4}\left[R_{2}^{2} \ln R_{2}-R_{1}^{2} \ln R_{1}+\frac{1}{2}\left(R_{2}^{2}-R_{1}^{2}\right)\right]+ \\
+\frac{E_{2}}{4}\left[R_{3}^{2} \ln R_{3}-R_{2}^{2} \ln R_{2}+\frac{1}{2}\left(R_{3}^{2}-R_{2}^{2}\right)\right] .
\end{gathered}
$$

After solving the linear equation system (26) the following formulas can be used for computing the stresses and displacements:

$$
\begin{gathered}
\sigma_{r 1}(r)=c_{1}+\frac{c_{2}}{r^{2}}+\frac{E_{1} C}{2} \ln r, \quad \sigma_{\varphi 1}(r)=c_{1}-\frac{c_{2}}{r^{2}}+\frac{E_{1} C}{2}(\ln r+1), \\
\sigma_{r 2}(r)=c_{2}+\frac{c_{3}}{r^{2}}+\frac{E_{2} C}{2} \ln r, \quad \sigma_{\varphi 2}(r)=c_{3}-\frac{c_{4}}{r^{2}}+\frac{E_{2} C}{2}(\ln r+1), \\
U_{1}(r)=\frac{1-\nu_{1}}{E_{1}} c_{1} r-\frac{1+\nu_{1}}{r E_{1}} c_{2}+\frac{C r}{2}\left[\left(1-\nu_{1}\right) \ln r-1\right]+\alpha_{1} T r, \\
U_{2}(r)=\frac{1-\nu_{2}}{E_{2}} c_{3} r-\frac{1+\nu_{2}}{r E_{2}} c_{4}+\frac{C r}{2}\left[\left(1-\nu_{2}\right) \ln r-1\right]+\alpha_{2} T r, \\
f_{1}=-U_{1}\left(R_{1}\right)=-\frac{1-\nu_{1}}{E_{1}} c_{1} R_{1}+\frac{1+\nu_{1}}{R_{1} E_{1}} c_{2}- \\
-\frac{C R_{1}}{2}\left[\left(1-\nu_{1}\right) \ln R_{1}-1\right]+\alpha_{1} T R_{1} \\
f_{2}=f_{3}=0, \\
u_{i}(r)=U_{i}(r)-U_{1}\left(R_{1}\right) \cos \varphi, \\
v_{i}(r)=C r \varphi+U_{1}\left(R_{1}\right) \sin \varphi, \quad(i=1,2),
\end{gathered}
$$


D. Gönczi

\section{SOLUTION IN THE FRAMEWORK OF STRENGTH OF MATERIALS}

On the basis of paper [13] by Ecsedi and Dluli it will be assumed that

$$
u=U(\varphi) \quad \text { and } \quad v=r \phi(\varphi)+\frac{\mathrm{d} U}{\mathrm{~d} \varphi}
$$

are the two displacement components for the whole two-layer composite curved beam. The strain-displacement relationships of the linearized theory of elasticity yield

$$
\begin{gathered}
\varepsilon_{r}=\varepsilon_{z}=\gamma_{r \varphi}=\gamma_{\varphi z}=\gamma_{r z}=0, \\
\varepsilon_{\varphi}=\frac{1}{r}\left(U+\frac{\mathrm{d}^{2} U}{\mathrm{~d} \varphi^{2}}\right)+\frac{\mathrm{d} \phi}{\mathrm{d} \varphi}
\end{gathered}
$$

which means that only one strain component is different from zero. Equations . (37) and (38) show that the displacements given by (36) satisfy the requirements of EulerBernoulli beam theory. The constitutive relation is the simple Hook's law applied here by taking into account the thermal effect:

$$
\sigma_{\varphi i}=E_{i} \varepsilon_{\varphi}-E_{i} \alpha_{i} T=\frac{E_{i}}{r}\left(U+\frac{\mathrm{d}^{2} U}{\mathrm{~d} \varphi^{2}}\right)+E_{i} \frac{\mathrm{d} \phi}{\mathrm{d} \varphi}-E_{i} \alpha_{i} T, \quad(i=1,2) .
$$

The stress resultant and the moment resultant of the stresses (the axial force and the bending moment) vanish since there is no mechanical load on the structure (Figure 2). Hence

$$
\begin{gathered}
N=\int_{R_{1}}^{R_{3}} \sigma_{\varphi} \mathrm{d} r=k_{1}\left(U+\frac{\mathrm{d}^{2} U}{\mathrm{~d} \varphi^{2}}\right)+k_{2} \frac{\mathrm{d} \phi}{\mathrm{d} \varphi}-\beta T=0, \\
M=\int_{R_{1}}^{R_{3}} r \sigma_{\varphi} \mathrm{d} d r=k_{2}\left(U+\frac{d^{2} U}{\mathrm{~d} \varphi^{2}}\right)+k_{3} \frac{\mathrm{d} \phi}{\mathrm{d} \varphi}-\gamma T=0 .
\end{gathered}
$$

where

$$
\left.\begin{array}{c}
k_{1}=E_{1} \ln \frac{R_{2}}{R_{1}}+E_{2} \ln \frac{R_{3}}{R_{2}}, \quad k_{2}=E_{1}\left(R_{2}-R_{1}\right)+E_{2}\left(R_{3}-R_{2}\right), \\
k_{3}=\frac{E_{1}}{2}\left(R_{2}^{2}-R_{1}^{2}\right)+\frac{E_{2}}{2}\left(R_{3}^{2}-R_{2}^{2}\right), \\
\beta=\alpha_{1} E_{1}\left(R_{2}-R_{1}\right)+\alpha_{2} E_{2}\left(R_{3}-R_{2}\right), \\
\gamma=\frac{\alpha_{1} E_{1}}{2}\left(R_{2}^{2}-R_{1}^{2}\right)+\frac{\alpha_{2} E_{2}}{2}\left(R_{3}^{2}-R_{1}^{2}\right)
\end{array}\right\}
$$

It follows from equations 40 and 42 that

$$
U+\frac{\mathrm{d}^{2} U}{\mathrm{~d} \varphi^{2}}=\frac{\beta k_{3}-\gamma k_{2}}{k_{1} k_{3}-k_{2}^{2}} T, \quad \frac{\mathrm{d} \phi}{\mathrm{d} \varphi}=\frac{\gamma k_{1}-\beta k_{2}}{k_{1} k_{3}-k_{2}^{2}} T .
$$

Combining equation (39) and (44) leads to the following expression for the circumferential normal stresses

$$
\sigma_{\varphi i}=E_{i}\left\{\frac{1}{k_{1} k_{3}-k_{2}^{2}}\left(\frac{\beta k_{3}-\gamma k_{2}}{r}+\gamma k_{1}-\beta k_{2}\right)-\alpha_{i}\right\} T, \quad(i=1,2) .
$$


With $\sigma_{\varphi i}$ the normal stress $\sigma_{r i}$ can be determined by utilizing the equilibrium equation

$$
\frac{\mathrm{d}}{\mathrm{d} r}\left(r \sigma_{r i}\right)=\sigma_{\varphi i} \quad(i=1,2)
$$

Substituting $\sigma_{\varphi i}$ and solving the resulting equation for $\sigma_{r i}$ yields

$$
\begin{aligned}
& \sigma_{r 1}(r)=E_{1}\left\{\frac{1}{\left(k_{1} k_{3}-k_{2}^{2}\right) r}\left(\beta k_{3}-\gamma k_{2}\right) \ln \frac{r}{R_{1}}+\right. \\
& \left.+\left(\gamma k_{1}-\beta k_{2}\right)\left(r-R_{1}\right)-\alpha_{1} \frac{r-R_{1}}{r}\right\} T, \quad R_{1} \leq r \leq R_{2}, \\
& \sigma_{r 2}(r)=\frac{R_{2}}{r} \sigma_{r 1}\left(R_{2}\right)+E_{2}\left\{\frac{1}{\left(k_{1} k_{3}-k_{2}^{2}\right) r}\left(\beta k_{3}-\gamma k_{2}\right) \ln \frac{r}{R_{2}}+\right. \\
& \left.+\left(\gamma k_{1}-\beta k_{2}\right)\left(r-R_{2}\right)-\alpha_{2} \frac{r-R_{2}}{r}\right\} T, \quad R_{2} \leq r \leq R_{3} .
\end{aligned}
$$

Integration of equation (44) by taking boundary condition (8) into account leads to the following expressions for the radial and circumferential displacements:

$$
\begin{gathered}
U(\varphi)=\frac{\beta k_{3}-\gamma k_{2}}{k_{1} k_{3}-k_{2}^{2}}(1-\cos \varphi) T, \\
v(\varphi, r)=\frac{\left(\gamma k_{1}-\beta k_{2}\right) r \varphi-\left(\beta k_{3}-\gamma k_{2}\right) \sin \varphi}{k_{1} k_{3}-k_{2}^{2}} T .
\end{gathered}
$$

\section{NumERicAl EXAMPLE}

The following data are used in the numerical example:

$$
\begin{gathered}
R_{1}=0.35 \mathrm{~m}, \mathrm{R}_{2}=0.4 \mathrm{~m}, \mathrm{R}_{3}=0.45 \mathrm{~m}, \\
E_{1}=200 \mathrm{GPa}, \mathrm{E}_{2}=70 \mathrm{GPa} \\
\nu_{1}=0.27, \nu_{2}=0.33 \\
\alpha_{1}=11 \cdot 10^{-6} \frac{1}{\mathrm{~K}}, \alpha_{2}=23 \cdot 10^{-6} \frac{1}{\mathrm{~K}} \\
T=200 \mathrm{~K}, \vartheta=\frac{3}{2} \pi .
\end{gathered}
$$

Beam component 1 is made of steel. The material of beam component 2 is aluminum. The graphs in Figures 2 and 3 show the normal stresses $\sigma_{r}$ and $\sigma_{\varphi}$ in a comparison with finite element solutions. The latter was carried out by using the commercial Abaqus CAE software (coupled temperature-displacement solver). 


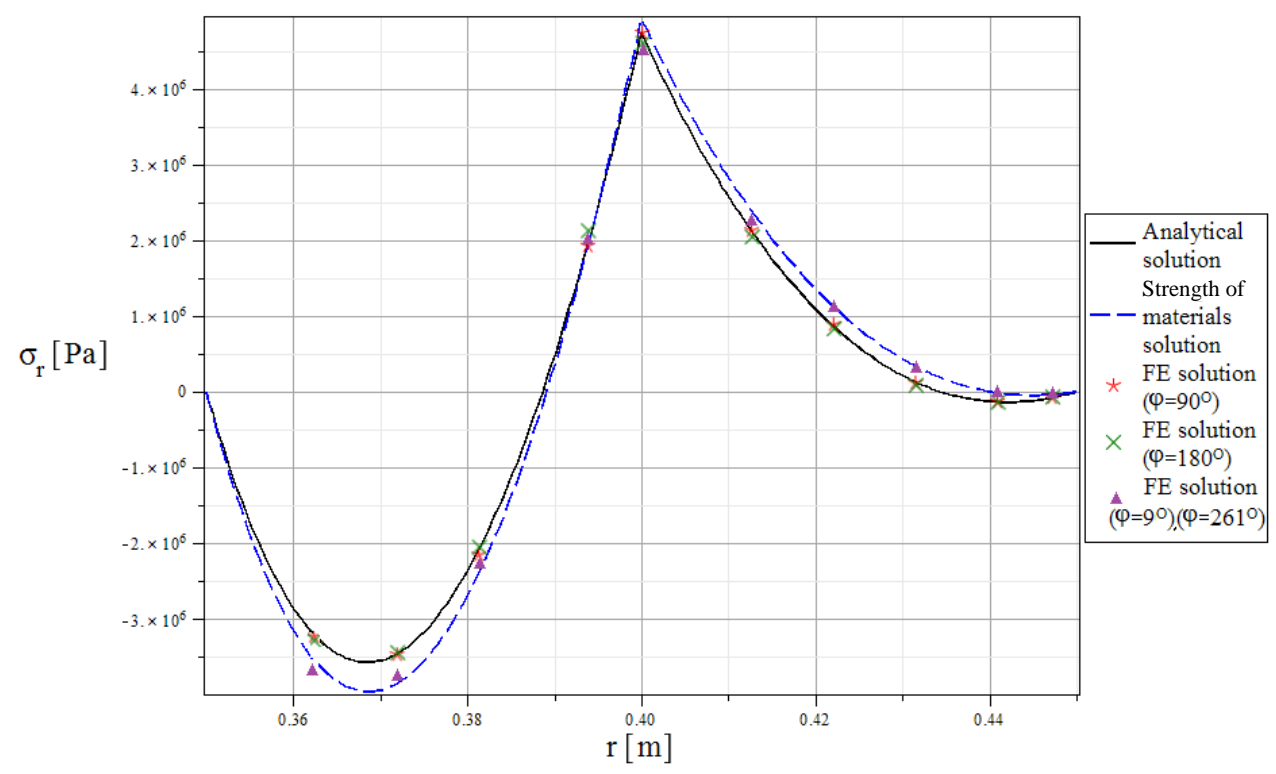

Figure 2. Plots of the radial normal stresses

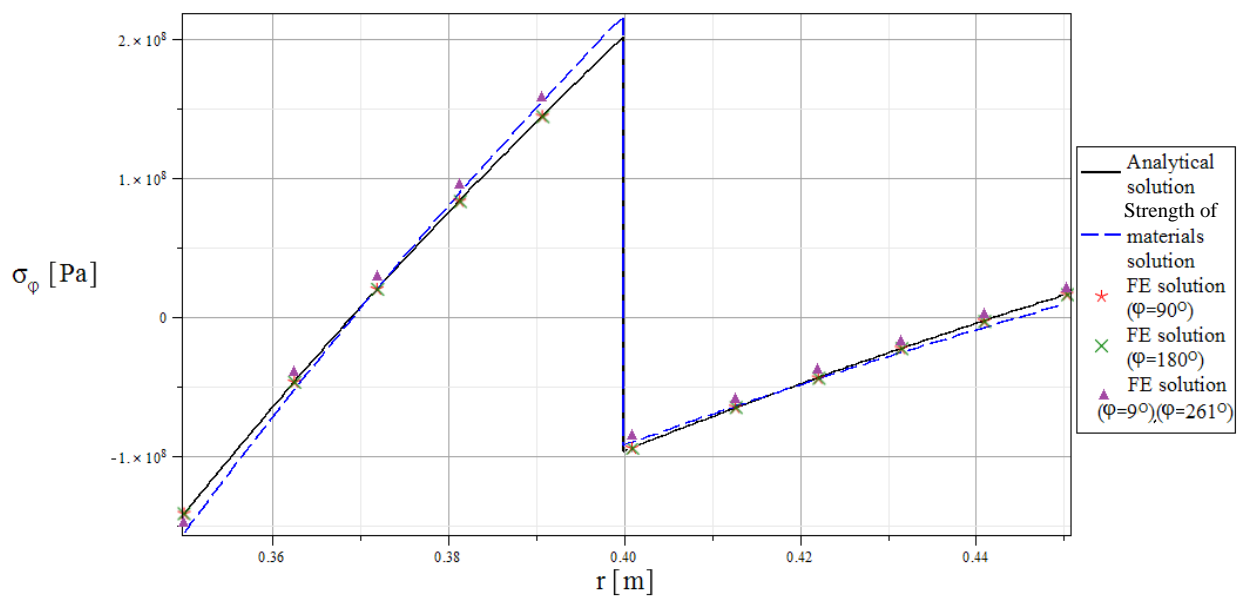

Figure 3. Plots of the circumferential stresses

In Figure 4 the finite element model of the bimetallic curved beam and von Mises equivalent stress field are presented, in which we can see that the stresses do not depend on the polar angle $\varphi$ aside from the narrow neighborhood of the end cross sections. 


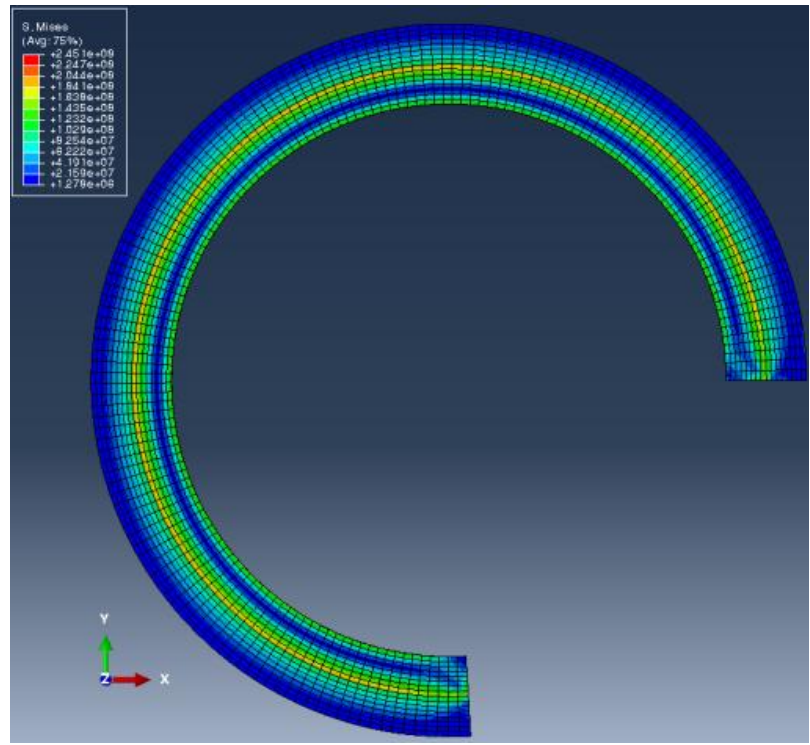

Figure 4. The finite element model with the equivalent stress field

Figures 5 and 6 show the 3D graphs of displacements $U(r, \varphi)$ and $v(r, \varphi)$ obtained by the previously presented elasticity solution when $R_{1} \leq r \leq R_{3}$ and $0 \leq \varphi \leq \pi$.

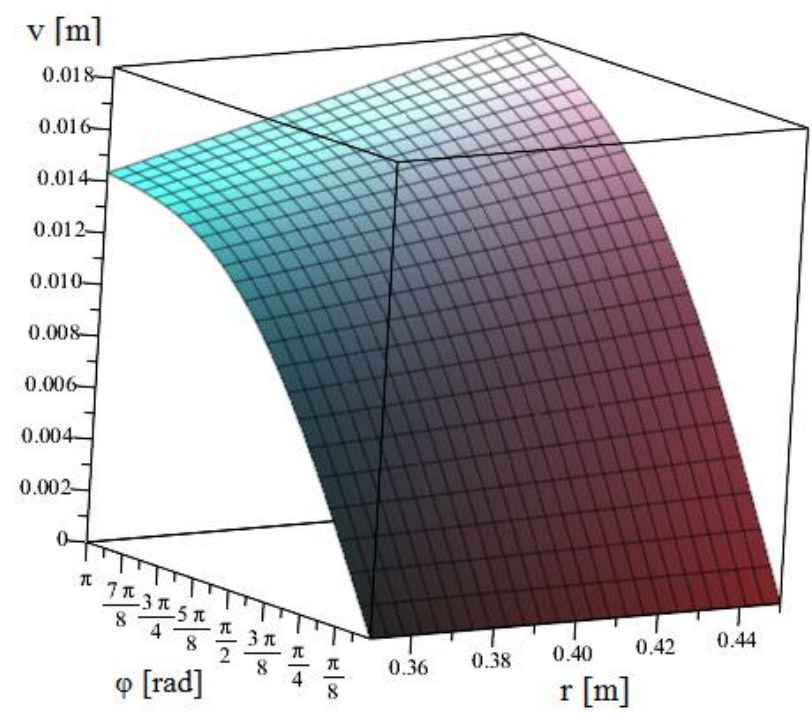

Figure 5. The circumferential displacement field 


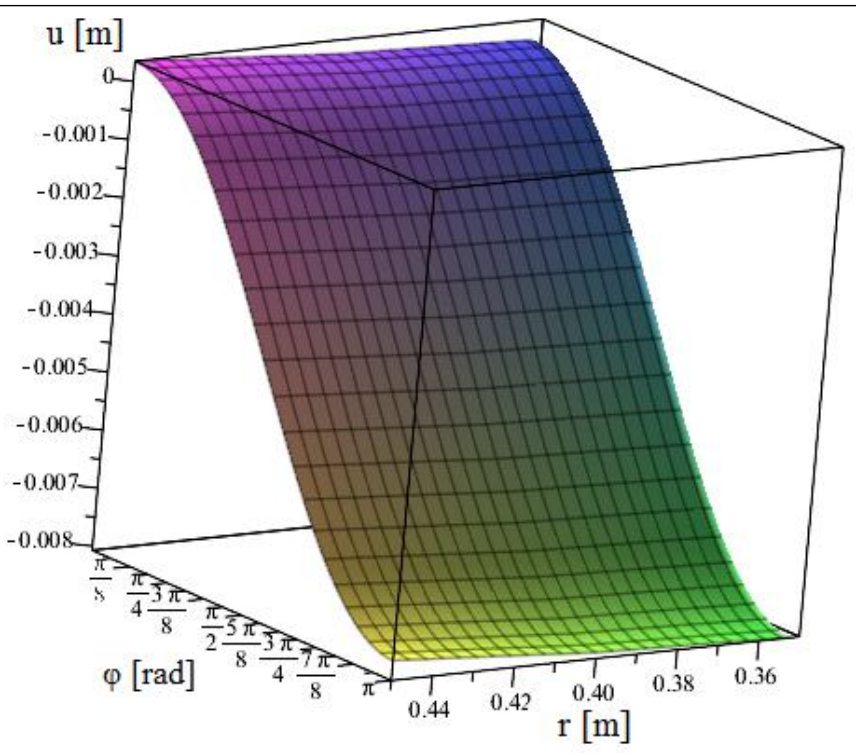

Figure 6. The radial displacement field

\section{Conclusions}

This paper presents two models to analyze the curved bimetallic beams. Presented elasticity and strength of materials approaches are checked by finite element simulations (using Abaqus FE software). The stresses obtained by the analytical method and FEM solution are in good agreement except for the end cross sections of the curved bimetallic beam. The presented analytical methods can be used to design a two-layered curved composite members which are in a high temperature environment.

\section{REFERENCES}

1. S. P. Timoshenko. "Analysis of bi-metal thermostats." Journal of the Optical Society of America, 11(3), (1925), pp. 233-255.

2. S. P. Timohsenko. The Collected Papers. McGraw Hill, New York, 1953. DoI: 10.1126/science.120.3113.339-a.

3. W. C. Young and Budynas R. G. Roark's Formulas of Stress and Strain. 7th ed. McGraw Hill, New York, 2002.

4. M. Aignatoaie. "FEA study on the elastic deformation process of a simple bimetal beam." Applied Mechanics and Materials, 371(8), (2013), pp. 448-452. DOI: $10.4028 /$ wWw . scientific.net/AMM.371.448.

5. E. Suhir. "Interfacial stresses in bimetal thermostats." Journal of Applied Mechanics, 56(3), (2013), pp. 595-600. DOI: 10.1115/1.3176133.

6. D. Ramos, I. Mertens, M. Callega, and I. Tamayo. "Study on the origin of bending induced by bimetallic effect on microcantilever." Sensors, 7(9), (2007), pp. 1757-1765. DOI: $10.3390 /$ s7091757. 
7. A. V. Rao, K. S. V. Prasad, M. Avinash, K. Nagababu, V. Manohar, P. S. R. Raju, and G. R. Chandra. "A study on deflection of a bimetallic beam under thermal loading using finite element analysis." International Journal of Engineering and Advanced Technology, 2(1), (2012), pp. 81-82.

8. W. H. Chu, M. Mehregany, and R. L. Mullen. "Analysis of tip deflection and force of a bimetallic cantilever microactuator." Journal of Micromechanics and Microengineering, 3(1), (1993), pp. 4-7. DOI: 10.1088/0960-1317/3/1/002.

9. B. A. Boley and I. H. Weiner. Theory of Thermal Stresses. Dover Publication, New York, 1997.

10. R.B. Hetnarski and Eslami M.R. Thermal Stresses - Advanced Theory and Application. Springer, Berlin, 2010. DOI: 10.1007/978-1-4020-9247-3.

11. Á. Lengyel and Ecsedi I. "Curved composite beam with interlayer slip loaded by radial load." Curved and Layered Structures, 2(1), (2015), pp. 50-58. DOI: 10.1515/cls-2015-0004

12. L.P. Kiss and G. Szeidl. "Nonlinear in-plane stability of heterogeneous circular beams subjected to a radial force at the crown point." Technische Mechanik, 35(1), (2015), pp. 1-30.

13. I. Ecsedi and K. Dluhi. "A linear model for the static and dynamic analysis of non-homogeneous curved beams." Applied Mathematical Modelling, 29(12), (2015), pp. 1211-1231. DOI: 10.1016/j.apm.2005.03.006 